Europhys. Lett., 51 (2), pp. 223-229 (2000)

\title{
Cluster persistence: A discriminating probe of soap froth dynamics
}

\author{
W. Y. Tam ${ }^{1}$, A. D. Rutenberg ${ }^{2}$, B. P. Vollmayr-Lee ${ }^{3}$ and K. Y. Szeto $^{1}$ \\ 1 Department of Physics, The Hong Kong University of Science and Technology \\ Clear Water Bay, Kowloon, Hong Kong \\ 2 Physics Department, McGill University - Montréal QC, Canada H3A 2T8 \\ 3 Department of Physics, Bucknell University - Lewisburg, PA 17837, USA
}

(received 25 October 1999; accepted in final form 18 May 2000)

PACS. 82.70.Rr - Aerosols and foams.

PACS. 05.70.Ln - Nonequilibrium and irreversible thermodynamics.

\begin{abstract}
The persistent decay of bubble clusters in coarsening two-dimensional soap froths is measured experimentally as a function of cluster volume fraction. A dramatically stronger decay is observed in comparison to soap froth models and to measurements and calculations of persistence in other systems. The fraction of individual bubbles that contain any persistent area also decays, implying significant bubble motion and suggesting that $\mathrm{T} 1$ processes play an important role in froth persistence.
\end{abstract}

Cellular structures are common in nature [1-3], and are studied in soap froths, polycrystallites, Potts models, biological cell clusters, and magnetic bubble arrays. They are composed of cells with varying sizes but identical composition, packed tightly against neighbors so that cell boundaries meet at vertices. In most cases, this structure is dynamic, i.e., part of an ongoing non-equilibrium process, and the challenge is to understand the role of dynamics in determining the structure. Individual cells continually evolve so as to reduce the total interfacial area in the system, which results in coarsening, or growth of the characteristic bubble size. "Topological" changes of cell adjacencies occur when neighbors swap (T1 process) and when cells are destroyed (T2 process) [4]. Topological transitions are effectively instantaneous in soap froths, while bubble areas grow smoothly. This is in contrast to polycrystallites or dynamical Potts models where topological processes occur at the same slow time scales as the evolution of bubble areas. Rapid topological transitions in soap froths allow us, in principle, to isolate the effects of these processes.

Soap froths are ideal systems to study cellular pattern evolution, since they coarsen at laboratory time scales and their time-dependent structure can be directly imaged in twodimensions $(2 d)$ with video microscopy [5]. In this paper, we study isotropic froths in $2 d$. We limit ourselves to the scaling regime [6] at late times, when the absence of non-universal transients facilitates both the analysis and the comparison with model systems.

A relatively new and general probe of non-equilibrium dynamics is the local power law decay of persistence towards zero [7], $P(t) \sim t^{-\theta}$, which in froths measures the fraction of the (c) EDP Sciences 
system that has always remained within the same bubble from time $t_{0}$ through to a later time $t \gg t_{0}$. A simple theoretical argument provides a lower bound $\theta \geq 1$ : the area of remaining bubbles in a coarsening froth increases linearly in time, implying that the number of bubbles $N \sim 1 / t[2,3]$. Since persistent volume cannot increase within a given bubble, then $P$ is bounded above by $N \sim 1 / t$ asymptotically. Numerical studies of $2 d$ cellular systems have found $\theta \leq 1[8-10]$, and are taken in conjunction with this theoretical bound to indicate $\theta=1$ asymptotically [10]. However, experimental studies of $2 d$ soap froths found $\theta>1$ [11], in clear disagreement, though consistent with the theoretical bound of $\theta \geq 1$. Evidently, persistence probes interesting physics in soap froths.

Most studies of froths use measures specific to the cellular topology, such as the distribution of the number vertices per cell. While such measures are important (see, e.g., [12]), it is also useful to develop probes of structure that are not unique to cellular systems. Results can then be compared to corresponding systems that have no cellular structure and hence no topological processes, so that the distinct effects of topological dynamics can be revealed. For example, it is interesting to compare froths with non-conserved scalar coarsening since they both exhibit curvature-driven interface motion while the latter has no topological processes. However, the choices for such probes are limited, since froth correlations generally reflect the cellular structure (see, e.g., [13]) independently of the dynamics.

Two of us have proposed a more general definition of persistence that makes use of the soap froth structure to create a "non-topological" measure that can be compared to other systems $[14,15]$. One first randomly selects a fraction $\phi$ of all of the bubbles at an initial time $t_{0}$ and colors them "green". One then asks what fraction of the system has always been green between $t_{0}$ and $t$. Essentially, we first make a virtual phase and then study its persistence properties. This allows us to fingerprint the dynamics at different length scales, as probed by the size of clusters of the virtual phase. The limit $\phi=0^{+}$consists of isolated bubbles whose persistence decays with the encroachment of any neighbor, which corresponds to the persistent decay previously studied in froths. Phases with $\phi>0$ have larger clusters, and for sufficiently large $\phi$ these clusters percolate through the system. Since $\phi$ is also the volume fraction, this "cluster persistence" can be directly compared to persistence in a two-phase system at the same volume fraction but lacking an underlying cellular structure.

Our primary results reported in this paper include i) experimental measurements of cluster persistence for all volume fractions $0<\phi \leq 1$ in a coarsening $2 d$ soap froth; for all $\phi$ we find a power law decay with exponent $\theta(\phi)$; ii) these exponents $\theta(\phi)$ are greater at corresponding $\phi$ than any other studied system with curvature-driven growth, cellular or non-cellular; iii) the persistence exponent is almost linear with $\phi$, for which we provide a mean-field argument; and iv) as $\phi \rightarrow 1, \theta(\phi)$ vanishes linearly with a considerably larger slope than predicted [14] for a mean-field droplet model without cellular structure. We interpret these results, particularly point ii), as evidence of the strong role of $\mathrm{T} 1$ processes in froths.

The experimental setup has been reported earlier [11,17]. We start our experiment by pumping approximately 20000 soap bubbles into a chamber formed by two rigid parallel plates $\left(26.7 \times 36.8 \mathrm{~cm}^{2}\right.$ with a $0.16 \mathrm{~cm}$ gap $)$, making an initially random froth that proceeds to coarsen. The froth was well drained before starting the experiment. Images of the froth were captured using a high-resolution CCD camera $(1037 \times 1344$ pixels $)$ every $10 \mathrm{~min}$, sufficient to follow the evolution of individual bubbles. (The entire chamber is slightly larger than the imaged region. We also excluded strips of bubbles near the edge of our image region to test boundary effects: none were found.) The scaling regime, as identified by a stationary distribution of bubble topologies, set in from $6 \mathrm{~h}$ when there were approximately 6000 bubbles, and we continued taking data until $160 \mathrm{~h}$ when 200 bubbles remained. Our persistence data was taken from $t_{0}=12 \mathrm{~h}$, with 2000 bubbles. Quantitatively consistent results, though noisier, 
are obtained using $t_{0}=26 \mathrm{~h}$.

We analyze the CCD images to identify each pixel with a given bubble (see [17]). Boundary pixels, on bubble boundaries, are randomly assigned to adjacent bubbles. For a given subset $\mathcal{S}$ of bubbles, we represent the pixels in $\mathcal{S}$ by a function $p_{\mathcal{S}}(j, t)=1$, where $j \in[1,1393728]$ counts the $M$ pixels, and for pixels outside of that subset of bubbles, $p_{\mathcal{S}}(j, t)=0$. The cluster persistence of the set $\mathcal{S}$ is then formally

$$
P_{\mathcal{S}}(t)=\frac{1}{M} \sum_{j=1}^{M} \prod_{t^{\prime}=t_{0}}^{t} p_{\mathcal{S}}\left(j, t^{\prime}\right),
$$

where $0 \leq P_{\mathcal{S}} \leq 1$. In other words, a pixel counts towards the persistence if it has always been in any of the bubbles belonging to $\mathcal{S}$. With this definition, the traditional measure of persistence corresponds to the case where $\mathcal{S}$ consists of a single bubble. More generally we can take arbitrary subsets of all of the $N\left(t_{0}\right)$ bubbles, where there are $2^{N\left(t_{0}\right)}$ possible subsets. We sample subsets by randomly choosing $m=\phi N\left(t_{0}\right)$ bubbles at a time. We fit the persistent decay for individual subsets, and then average the resulting exponents - binning them with respect to $\phi(\mathcal{S})$. For each subset, we use the time-average of $\phi(\mathcal{S})$. The resulting persistence decay is characterized by a power law

$$
P_{\phi}(t) \sim t^{-\theta(\phi)} \sim\langle A\rangle^{-\theta^{\prime}(\phi)} .
$$

We expect $\theta^{\prime}=\theta$, since the average bubble area in the froth, $\langle A\rangle$, is asymptotically proportional to $t$ [3], but we measure $\theta^{\prime}$ to eliminate the dependence on the absolute time origin and to minimize transient effects $[10,17]$. The decay is well fit to a power law, as illustrated for five subsets on the left of fig. 1, and we plot all of the resulting exponents on the right of fig. 1 as a function of volume fraction, $\phi$. The errors shown are from the statistical variation between subsets at a given volume fraction, and can be reduced with more sampling. We selected about 1000 multiple-bubble subsets out of the $2^{2000}$ possibilities, in addition to the 2000 single-bubble subsets.

On the right of fig. 1 we plot our data for $\theta(\phi)$ as open circles, and include a quadratic fit as a solid curve (described below). For comparison, we include data from previous studies of corresponding systems with curvature-driven growth and volume fraction $\phi$. These include the persistence of one phase of the $p$-state Potts model, with $\phi=1 / p$, simulated $[8,9]$ and plotted as triangles for $0<\phi \leq 1 / 2$. Potts systems, particularly when $p \rightarrow \infty$, have been proposed as soap froth analogues [18]. The Potts data fits relatively well on a continuous curve (dotted line), interpolating smoothly between $p=2$ with a two-phase non-cellular structure, $3<p<\infty$ with a cellular structure and some bubble coalescence, and $p=\infty$ with a cellular structure and no bubble coalescence. Also shown (open square) is a previous persistence experiment on twisted nematic liquid crystals [19], an Ising model analog with $\phi=1 / 2$. Finally, an analytic result for a mean-field droplet model, valid for $\phi \approx 1$, describing non-conserved order parameter dynamics with a time-dependent applied field is plotted as a dashed line [14]. We note that remarkable agreement is seen between the Potts data, the experimental work on twisted nematics, and the extrapolation of the $O(1-\phi)$ exact result for coarsening droplets. Neither the nematic nor the droplet system have cellular structures, and the relatively good agreement between them and the Potts data indicates that persistence is not greatly affected by the cellular structure per se. Nevertheless, we find froth decay exponents that are dramatically larger than all these corresponding systems!

Qualitatively, the froth persistence data is strikingly linear. The droplet model studied in ref. [14] used a mean-field treatment, asymptotically correct as $\phi \rightarrow 1$, to obtain the linear 

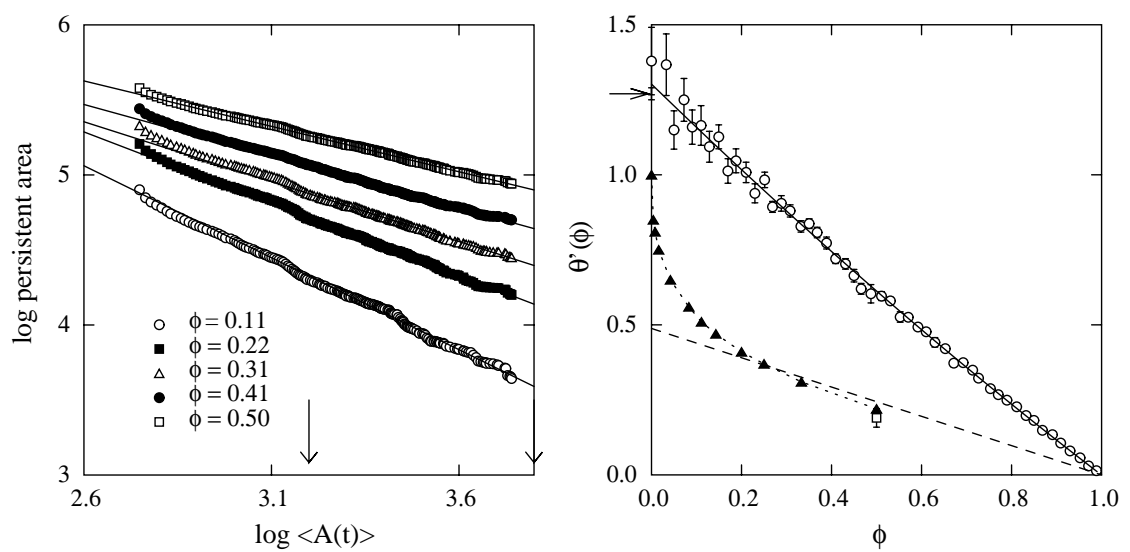

Fig. 1 - Left: $\log$-log (base 10) plot of cluster persistence decay for various volume fractions $\phi$. The arrows indicate the fitting region used to extract all persistence exponents. Right: persistence decay exponent as a function of volume fraction for our soap froth data (open circles), Potts models with $\phi=1 / p$ (triangles), twisted nematics (open square), and droplet calculation (dashed line). The solid curve is a quadratic fit (see text), while the dotted curve is a guide to the eye. The arrow indicates the $\phi=0$ value expected from bubble motion, as probed by the decaying number of bubbles with persistent cores (see text and fig. 2).

variation $\theta(\phi) \simeq 0.48797(1-\phi)$. This motivates a similar mean-field argument for froths. In essence, if the local environment around a persistent region is entirely random, then the rate of persistent volume lost from a subset of bubbles $\mathcal{S}$ of volume fraction $\phi$ is given simply by the loss of persistent volume from individual bubbles times the chance, $1-\phi$, that it is being lost to a bubble outside of $\mathcal{S}$. That is, $\dot{P}_{\phi} / P_{\phi}=(1-\phi) \dot{P}_{0} / P_{0}$, where $P_{\phi}$ is the cluster persistence, and $P_{0}$ is the $\phi \rightarrow 0^{+}$limit of single bubbles. This equation would result in a decay exponent $\theta(\phi)=\theta(0)(1-\phi)$. It is a priori unlikely that this drastic mean-field approximation, or the resultant linearity, holds as an exact relationship. For example, in the droplet model [14] appreciable curvature corrections, expected to enter at $O\left[(1-\phi)^{2}\right]$, must develop since $\theta(0)=1$. Indeed, we see below that such non-mean-field effects occur in soap froths. However, the near linearity of the froth data does imply that correlation effects are small for cluster persistence in soap froths.

To fit all of our froth data, we use an expansion in powers of $1-\phi$, making no assumptions about the behavior near $\phi=1$, and obtain an excellent fit: $\theta(\phi)=-0.001^{ \pm 0.003}+1.15^{ \pm 0.02}(1-$ $\phi)+0.15^{ \pm 0.03}(1-\phi)^{2}$. Higher powers in $(1-\phi)$ are not indicated, since we find that the coefficients are zero within error bars. The quadratic term is small but significantly non-zero - this upward curving $\theta(\phi)$ indicates that the persistence decays, for all $0<\phi<1$, slightly slower than our mean-field argument would predict. Evidently cluster persistence reveals positive correlations between persistent regions and neighboring cells of the same phase. The variation near $\phi=1$ is linear within errors, as was conjectured in [14] by analogy with the droplet model.

We turn now to the question of the strong persistence decay in froths relative to other curvature-driven systems, for which purpose we consider the standard persistence exponent, or equivalently the persistence of isolated bubbles. The extrapolation to $\phi=0$ of our quadratic fit gives an improved value of $\theta(0)=1.30 \pm 0.01$ for single bubbles [20], which significantly disagrees with previous predictions of $\theta(0)=1$. In the scaling state, if a constant fraction 

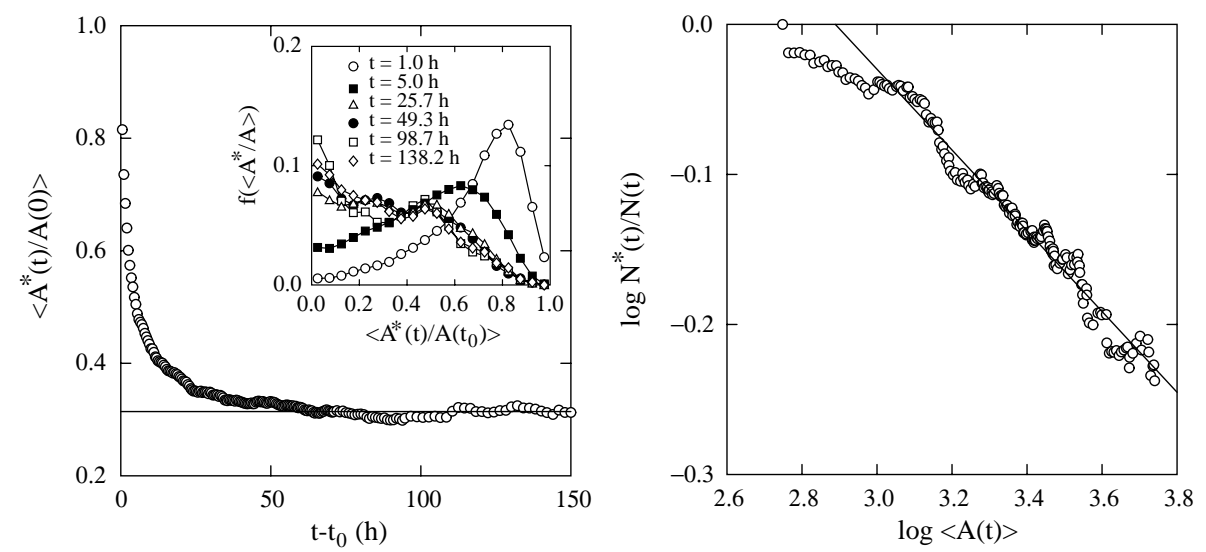

Fig. 2 - Left: plot vs. time of $\left\langle A^{*}(t) / A\left(t_{0}\right)\right\rangle$, the average of the persistent area within a bubble at time $t$ normalized by the entire area of the same bubble at $t_{0}$. The averages are restricted to those bubbles with non-zero persistent regions. The horizontal line, at $\left\langle A^{*}(t) / A\left(t_{0}\right)\right\rangle=0.314$, is the average value from 50 to $150 \mathrm{~h}$. The distribution for various times is shown in the inset; it approaches a stationary form. Right: fraction of bubbles that contain persistent volume at time $t v s$. the average bubble area. The best fit (solid line) gives a decay exponent $-0.27 \pm 0.02$, where the error bar is approximate.

of remaining bubbles have persistent cores, and if these cores have a non-decaying average area, then the persistence is proportional to the density, and $\theta=1$. Our result of $\theta \simeq 1.3$ implies either a continual erosion of the persistent cores in bubbles, a continual decrease in the fraction of bubbles with persistent cores, or both. By direct measurement we find that the average persistent core size does not decay at late times, while in contrast, the fraction of bubbles with persistent cores steadily decays and entirely accounts for the exceptionally strong persistence decay of individual bubbles in soap froths. This strong decay is not shared by solutions of topological or droplet mean-field models (with or without vertices, respectively) or by simulations of Potts models.

We first measure the average persistent area $\left\langle A^{*}(t) / A\left(t_{0}\right)\right\rangle$, where averages are restricted to the $N^{*}(t)$ bubbles that contain persistent regions at time $t$, and we have normalized each bubble by its area at $t_{0}$. As shown on the left in fig. 2, it does not change in time after $50 \mathrm{~h}$, and its distribution approaches a time-independent form. This is qualitatively similar to the evolution of dilute droplet models [14], where $\left\langle A^{*}(t) / A\left(t_{0}\right)\right\rangle \approx 1$ since all surviving drops have a persistent core from $t_{0}$.

We next measure the number of bubbles that contain any persistent area, $N^{*}(t)$, and plot the ratio $N^{*} / N$ vs. $\langle A(t)\rangle$ on the right in fig. 2. While noisy, there is a clear decay, with a best fit giving $N^{*} \sim\langle A\rangle^{-1.27 \pm 0.02}$. This is a dramatic and unexpected result. It implies a new length scale $L^{*} \sim t^{0.64 \pm 0.01}$, characterizing the separation of bubbles that have persistent volume, that grows faster than the bubble size $L \sim t^{1 / 2}[2,3]$. It also independently implies a persistence decay $P_{0}^{*}=\left\langle A^{*}(t)\right\rangle N^{*} \sim N^{*}$, indicated by the arrow in fig. 1, that is consistent with our previous result.

The power law decay of $N^{*}(t) / N(t)$ implies significant movement of the center of mass of a bubble, since persistence within a given surviving bubble cannot be entirely lost without the bubble moving off of its original center of mass. Bubble motion on the scale of the bubble size is therefore directly implicated in the strong persistence decay of soap froths.

It is worthwhile to speculate on the causes of the bubble motion. We have investigated 
the decay of persistence of individual bubbles, and found numerous discrete decreases in the persistent fraction correlated with significant and sudden motion of the bubble center of mass. This implicates the correspondingly sudden topological processes in bubble motion and hence in strong persistence decay. T2 processes do not lead to sudden bubble motion for three-sided bubbles as they vanish, and shrinking bubbles with four or five sides lose their excess sides through T1 processes before they vanish. As a result, T1 processes are the main source of bubble motion. Indeed, accurate numerical simulations of planar froths have seen that bubble motion extends appreciable distances from T1 events [21], in agreement with our observations. It had been thought that $\mathrm{T} 1$ processes had no discernible effect on foam structure due to the unchanged growth of bubble size when weak shear was applied [22]. However, the growth of bubble size is well predicted by mean-field-like arguments [3] that should become more accurate with shear due to mixing effects, and so is an incomplete probe of structure. In contrast, our results on persistence decay show that $\mathrm{T} 1$ processes, often not included in theoretical work [23], are important in the understanding of soap froth evolution. This is consistent with results of numerical modeling that show that the width of the distribution of bubble sides, $\mu_{2}$, is dependent on the specific implementation of $\mathrm{T} 1$ processes [24].

Potts models also have a cellular structure and hence topological processes occur throughout their evolution. There, within numerical accuracy $\theta^{\prime}(0)=1[8]$, and so we must have both $\left\langle A^{*}\right\rangle$ and $N^{*} / N$ asymptotically constant and hence no significant motion of bubbles. This follows from the monotonicity of $\left\langle A^{*}(t)\right\rangle$ and $N \sim 1 /\langle A\rangle \sim 1 / t$. What leads to this distinction between soap froths and $p \rightarrow \infty$ Potts models? Qualitatively, topological processes occur much more slowly in Potts models as compared with soap froths [2]. However, the relative rate of $\mathrm{T} 1$ and $\mathrm{T} 2$ processes in Potts model evolution has neither been investigated nor compared with soap froths, where the ratio of T1 :: T2 is approximately $3:: 2$ [21]. As yet, it is impossible to distinguish the role of the frequency of topological $\mathrm{T} 1$ processes from their speed in the strong decay of persistence.

In summary, from $2 d$ experiments of coarsening soap froths, we have measured persistence decay exponents as a function of volume fraction. We find that soap froths exhibit much faster persistence decay at all volume fractions compared to other systems, including existing models of soap froth evolution in which persistence has been measured. Over time, a decreasing fraction of surviving bubbles contain any persistent area at all. This implies significant motion of bubbles that is strongly correlated with topological processes in the froth.

While a direct study of $\mathrm{T} 1$ processes is clearly desirable, we have demonstrated that the decay of persistence is a sensitive probe of the effect of T1 processes. More fundamentally, persistence decay also allows the comparison of different cellular systems, for instance between planar and bulk soap froths. In light of the possible proliferation of $\mathrm{T} 1$ processes in the latetime regime of bulk $3 d$ froths [25], it would be interesting to analyze persistence decay in those systems. Such an exercise is now possible, in principle, with the development of spatially resolved MRI techniques [26].

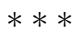

WYT and KYS are supported by the Competitive Earmarked Research Grant No. HKUST 6123/98P of the Research Grants Council of Hong Kong. ADR thanks the NSERC, and le Fonds pour la Formation de Chercheurs et l'Aide à la Recherche du Québec for financial support, and J. STAVANs for discussions and introductions. 


\section{REFERENCES}

[1] Weaire D. and Rivier N., Contemp. Phys., 25 (1984) 59.

[2] Glazier J. A. and Weaire D., J. Phys. Condens. Matter, 4 (1992) 1867.

[3] Stavans J., Rep. Prog. Phys., 56 (1993) 733.

[4] Levitan B. and Domany E., Int. J. Mod. Phys. B, 10 (1996) 3765.

[5] Glazier J. A., Gross S. and Stavans J., Phys. Rev. A, 36 (1987) 306.

[6] Stavans J., Phys. Rev. A, 42 (1990) 5049.

[7] Bray A. J., Derrida B. and Godrèche C., Europhys. Lett., 27 (1994) 175; Marcos-Martin M. et al., Physica A, 214 (1995) 396.

[8] Derrida B., de Oliveira P. M. C. and Stauffer D., Physica A, 224 (1996) 604; Hennecke M., Physica A, 252 (1998) 173. Note that in fig. 1 we use Hennecke's data for $p>2(\phi<1 / 2)$.

[9] Levitan B. and Domany E., Europhys. Lett., 38 (1997) 485.

[10] Hennecke M., Physica A, 246 (1997) 519. The previous values of $\theta<1$ are likely due to transients.

[11] TAM W. Y. et al., Phys. Rev. Lett., 78 (1997) 1588.

[12] Aste T., Szeto K. Y. and Tam W. Y., Phys. Rev. E, 54 (1996) 5482.

[13] Szeto K. Y., Aste T. and Tam W. Y., Phys. Rev. E, 58 (1998) 2656.

[14] Lee B. P. and Rutenberg A. D., Phys. Rev. Lett., 79, 4842 (1997). Note that $\phi=1-\epsilon$, where $\epsilon$ is the minority volume fraction in that paper.

[15] Our persistence approach is complementary to work on "survivors", or the statistics of the bubbles at an earlier time $t_{0}$ that will survive until a later time $t[16,17]$. The topological distribution of survivors is an exacting probe of soap froth models [4], and the area contained in the survivors at $t_{0}$ is an upper bound on the persistent area -recovering $\theta \geq 1$ [9].

[16] Levitan B. et al., Phys. Rev. Lett., 73 (1994) 756.

[17] Tam W. Y., Cheung K. M. and Szeto K. Y., Phys. Rev. E, 57 (1998) 7354.

[18] Glazier J. A., Anderson M. P. and Grest G. S., Phys. Mag. B, 62 (1990) 6115.

[19] Yurke B. et al., Phys. Rev. E, 56 (1997) 40.

[20] Previous work on the persistence decay of froths found $\theta^{\prime}(0)=1.16 \pm 0.02$ [11] by fitting the average of all of the single bubble decay curves. The resulting fit error is a significant underestimate. In this paper we extract error bars directly from the variances between different decay curves. For example, we directly measure $\theta^{\prime}(0)=1.38 \pm 0.11$, as shown in fig. 1 .

[21] Herdtle T. and Aref H., J. Fluid Mech., 241 (1992) 233.

[22] Gopal A. D. and Durian D. J., Phys. Rev. Lett., 75 (1995) 2610.

[23] Flyvbjerg H., Phys. Rev. E, 47 (1993) 4037; Physica A, 194 (1993) 298; Stavans J., Domany E. and Mukamel D., Europhys. Lett., 15 (1991) 479.

[24] Chae J. J. and Tabor M., Phys. Rev. E, 55 (1997) 598.

[25] Durian D. J., Weitz D. A. and Pine D. J., Phys. Rev. A, 44 (1991) R7902.

[26] Gonatas C. P. et al., Phys. Rev. Lett., 75 (1995) 573. 